\title{
Open
}

\section{"Is a cure in my sight?" Multi-stakeholder perspectives on phase I choroideremia gene transfer clinical trials}

\author{
Shelly Benjaminy, BSc, MSc' ${ }^{1}$ lan MacDonald, MDCM, MSc${ }^{2}$ and Tania Bubela, PhD, JD ${ }^{1}$
}

Purpose: Ocular gene transfer clinical trials are raising patient hopes for the treatment of choroideremia - a blinding degenerative retinopathy. Phase I choroideremia gene transfer trials necessitate communicating about the risks of harm and potential benefits with patients while avoiding the sensationalism that has historically undermined this field of translational medicine.

Methods: We conducted interviews between June 2011 and June 2012 with 6 choroideremia patient advocates, 20 patients, and 15 clinicians about their hopes for benefits, perceived risks of harm, and hopes for the time frame of clinical implementation of choroideremia gene transfer.

Results: Despite the safety focus of phase I trials, participants hoped for direct visual benefits with evident discrepancies between stakeholder perspectives about the degree of visual benefit. Clinicians and patient advocates were concerned by limited patient attention to risks of harm. Interviews revealed confusion about the time frames for the clinical implementation of choroideremia gene transfer and patient urgency to access gene transfer within a limited therapeutic window.

Conclusion: Differences in stakeholder perspectives about choroideremia gene transfer necessitate strategies that promote responsible communications about choroideremia gene transfer and aid in its translation. Strategies should counter historical sensationalism associated with gene transfer, promote informed consent, and honor patient hope while grounding communications in current clinical realities.

Genet Med advance online publication 26 September 2013

Key Words: choroideremia; gene therapy; gene transfer; informed consent; phase I

\section{INTRODUCTION}

Recent successes in gene transfer (GT) for ocular applications have revived long-standing hopes for the field. In the 1990s, GT was hailed as a "miracle technology" and was misleadingly referred to as "gene therapy" in spite of its experimental nature. Despite high hopes, serious setbacks damaged GT's reputation $^{2}$ and resulted in significant public scrutiny. In 2007, however, phase I trials for Leber congenital amaurosis (LCA), an infant-onset retinopathy, displayed short-term safety and efficacy. ${ }^{3-5}$ Optimistic clinicians described these early results as "a paradigm shift in our management of retinal dystrophies of all types, previously thought to be an untreatable group of human diseases".

While adults derived some visual benefits in the LCA trials (improvements in visual acuity, visual field, pupillary reaction, and nystagmus), ${ }^{3-5}$ children showed the most dramatic responses in later trials. Indeed, after GT, an 8-year-old child displayed comparable light sensitivity levels to age-matched controls, ${ }^{7}$ indicating that early GT application may lead to greater visual improvements. The results therefore suggested a limited therapeutic window for visual gain, a finding that has fueled patient urgency to access $\mathrm{GT}^{8}{ }^{8}$ especially as LCA trials are now entering phase III (clinicaltrials.gov NCT00999609). While concerns have recently been raised about the long-term efficacy of the intervention in the LCA trials, ${ }^{9}$ further earlystage GT trials for retinopathies now include Stargardt disease (clinicaltrials.gov NCT01367444), retinitis pigmentosa (clinicaltrials.gov NCT01482195), and Usher syndrome (clinicaltrials.gov NCT01505062). In October 2011, a phase I choroideremia trial was initiated in the United Kingdom focused on safety and dosage (clinicaltrials.gov NCT01461213). Choroideremia is a sex-linked retinopathy affecting $\sim 1$ of 50,000 males. In the absence of a treatment, choroideremia causes progressive vision loss from childhood to legal blindness by middle age. ${ }^{10}$ Two further trials are set to commence in North America.

Positive media coverage of ocular GT of early evidence from the LCA trials ${ }^{11}$ combined with optimistic statements by researchers and clinicians have ignited patient hopes for a successful treatment for a range of retinopathies. ${ }^{8}$ High hopes based on limited data, however, present challenges for communicating about risks of harm and potential benefits of early-stage trials as well as time frames for clinical application of therapies. Of greatest concern, communication deficits may compromise the integrity of informed consent for potential trial participants. ${ }^{12}$ Participants may misunderstand the safety focus of early-stage trials, expecting therapeutic benefit. Termed 'therapeutic misconception', patients commonly conflate the goals of research and those of clinical care. ${ }^{13}$ Similarly, patients may overestimate

${ }^{1}$ School of Public Health, University of Alberta, Edmonton Clinic Health Academy, Edmonton, Alberta, Canada; ${ }^{2}$ Department of Ophthalmology, University of Alberta, Royal Alexandra Hospital, Edmonton, Alberta, Canada. Correspondence: Tania Bubela (tbubela@ualberta.ca) 
therapeutic benefits or underestimate risks of harm associated with research, termed 'therapeutic misestimation. ${ }^{14}$ Therapeutic misconception and misestimation are not only characteristic of patients, but also affect stakeholders involved directly and indirectly in the informed consent process, such as clinical investigators and research oversight bodies. ${ }^{15}$

Despite the rapid advances in ocular GT, little research has been directed at exploring the values and priorities of key stakeholders such as patients, patient advocates, and clinicians. ${ }^{16}$ Similar research gaps are evident in the translation of other novel biotechnologies from bench research to clinical trials. ${ }^{17}$ Our study, therefore, addresses multistakeholder perspectives about ocular GT trials, focusing on choroideremia. It examines the commonalities and differences in views between stakeholder groups (patients, clinicians, and patient advocates). We specifically address the following issues: (i) potential benefits and therapeutic hopes; (ii) risks of harm; (iii) urgency to access GT; and (iv) time frames for clinical implementation. We conclude with recommendations for responsible communications for GT trials that counter the historical sensationalism associated with this field of biotechnology. ${ }^{18}$

\section{MATERIALS AND METHODS}

\section{Participants}

We interviewed 20 North American choroideremia patients (P); 15 clinicians from North America and Europe (C); and 6 representatives of North American and European patient advocacy organizations (advocates/A). Patients were affected males older than 18 years of age who may be eligible to participate in the upcoming choroideremia trials. Five of the 15 clinicians, ophthalmologists and genetic counselors who specialized in ocular genetics, were directly involved in ocular GT trials. Advocates were representatives from the boards of patient advocacy organizations involved in fundraising for choroideremia research and patient education. We recruited patients from the Regional Eye Centre, Royal Alexandra Hospital, Edmonton, and via notices on patient advocacy organization websites. We recruited clinicians and advocates via email and at conferences.

\section{Data collection}

S.B. conducted $45 \mathrm{~min}$ to $1 \mathrm{~h}$ semistructured interviews with each participant between June 2011 and June 2012. The interview guides were informed by research on communication in genetics ${ }^{15,19,20}$ and were reviewed for depth and breadth of coverage by experts in ocular genetics, risk communication, and bioethics. Interview guides were specific to stakeholder groups, but all addressed potential benefits, risks of harm, and time frames of choroideremia GT trials. Patient interviews focused on experiences living with choroideremia; understanding of diagnosis; awareness of ocular GT research; sources of communications about GT; and views about participation in CHM trials. Clinician interviews focused on diagnosis; management strategies for visual impairment; perspectives on patient hopes; and communication strategies with other stakeholders. Interviews with advocates focused on the supports and information they provided for members; position on GT; communication with members, including about GT; advocacy role; and external stakeholder communications strategies. The Health Panel of the Ethics Review Board at the University of Alberta approved this study.

\section{Data analysis}

We analyzed verbatim transcripts of digitally recorded interviews using NVivo 9.1 data analysis software (QSR international 2010). Our qualitative analysis of interview transcripts, using the 'constant comparison method', involved a rigorous and iterative process. ${ }^{21}$ S.B. coded each line of a subset of transcripts, and analyzed these codes for similarities and differences between transcripts. Starting with this close examination of the transcripts, and through ongoing discussions with other investigators, S.B. developed a preliminary codebook representative of key perspectives and ideas. S.B. and T.B. refined the codebook as S.B. analyzed the remaining transcripts and reexamined the already analyzed transcripts as new codes became apparent. Our coding method provided a rich analysis of the data as multiple codes could apply to different dimensions of the same text. As explained by Illes et al., ${ }^{17}$ the goal of such analysis is "to identify a broad range of perspectives, not necessarily a consensus among participants, and to deliver a coherent conceptual description of the data that capture thematic patterns and characterize the phenomena of interest while accounting for the individual variations within them."

Once transcript coding was completed, S.B. grouped codes into themes and subthemes. T.B. reviewed both the codes and the clustering of codes into key themes. A third coder external to the project reviewed the final codebook and transcripts to provide an objective assessment. The external coder suggested collapsing closely related themes, adding nuanced subthemes to emphasize key ideas, and refining the definitions of several constructs. Any disagreements were discussed and consensus was reached between all coders. Finally, S.B. revised transcript coding again in light of the final codebook. To ensure that we captured participant views accurately, S.B., I.M., and T.B. prepared reports explaining the main themes that emerged from the interviews. We sent these reports to participants, inviting them to ask additional questions or provide feedback. Responses from participants were overall supportive of the results and their interpretation. We also reviewed all selected quotes in the context of the original transcripts to ensure that they retained their meaning.

\section{Study limitations}

Our recruitment of patients from advocacy websites may have biased our sample to those more knowledgeable about their disease and GT, which may have influenced their risk perspectives and hopes. Choroideremia trials in North America have not yet started, limiting our patient interviews to potential research participants. Finally, we interviewed only a small number of advocates, who had the most heterogeneous perspectives of all stakeholders. However, these represented all of the advocacy organizations interested in choroideremia worldwide. 


\section{RESULTS}

\section{Potential benefits of choroideremia GT}

While stakeholders agreed on many potential benefits of GT, key differences arose in their visual outcome hopes along a continuum from no visual benefit to a complete cure. Patients described aspirational (to future patients or society), collateral (arising from research participation that do not depend on the experimental intervention such as empowerment secondary to research contribution), and direct benefits (associated with experimental intervention such as visual improvement) arising from participation in a GT trial (Table 1). ${ }^{22}$ Visual benefit was the main motivator for patients, even though most recognized the possibility that GT trials may provide no visual benefit. Clinician and advocate visual benefit perspectives, mediated by the outcomes of the LCA trials, ${ }^{3-5}$ converged from slowing down vision loss, halting vision loss, to a partial reversal of lost vision. These stakeholders emphasized that GT could provide a treatment but not a cure because GT is unlikely to provide regenerative benefits to retinal cells that have already degenerated. ${ }^{23}$ Patients, on the other hand, voiced hopes for a cure or a partial reversal of lost vision, but most hoped for a halt in vision loss.

Some clinicians raised patient difficulties in conceptualizing the meaning of a treatment for choroideremia in light of their understanding of "treatment" for other diseases: "People say therapy like: Oh, we can treat the pneumonia, therefore my lungs are normal again. I'm going to have gene therapy all my vision is going to be restored."-C11. Some clinicians also believed that patients lack the tools to distinguish between the potential visual outcomes of choroideremia GT. However, despite such clinician concerns, most patients articulated their hopes for visual outcome in a nuanced manner. Much like other stakeholders, most patients hoped for a treatment rather than a cure.

\section{Risks of harm of choroideremia GT}

Patients were focused on gaining access to GT rather than on risks of harm (Table 2). Clinicians explained that patients seldom inquired about the safety of GT and felt that patient hope for a treatment diverted attention from the risks of harm: "Deep down in the heart of any patient for whom there is no effective treatment or cure, clinical trials mean "this might be the thing that's going to help me"...so safety is...not their first concern."-C10.

Supporting this view, many patients questioned the interviewer about gaining access to CTs rather than about risks of harm. A quarter of patients even expressed a "no risk" perspective: "For me there's no risks. The only thing that could happen is to get some [vision] back."-P7. Most patients, however, acknowledged the risk of accelerated vision loss, but opinions diverged with respect to the personal relevance of this risk. Some patients were hesitant in risking their remaining functional vision. Others, especially those who described their visual field as significantly deteriorated, expressed a willingness to accept the risk of accelerated vision loss: "If I lose my sight, it's going anyway." $-\mathrm{P} 2$

Patients also described factors that attenuated their risk perspectives. Many patients normalized the nature of risk as inherent to all trials: "Accepting the risk would go with [a GT trial] and moving the scientific project forward, because you need people to...step forward and say I'm willing to do that."-P13. Others expressed trust in the clinicians, researchers, or scientific traditions behind GT trials: "I have faith in our medical system, that they do make sure that things are safe." - P8

\section{Urgency: "I would do anything to participate in a clinical trial" \\ Patients and patient advocates affected by choroideremia emphasized their urgency to access GT: "If [GT] came out}

Table 1 Potential benefits of participating in choroideremia gene transfer clinical trials described by patients

\begin{tabular}{|c|c|}
\hline Reason & Illustrative quotation \\
\hline \multicolumn{2}{|l|}{ Aspirational and collateral benefits } \\
\hline $\begin{array}{l}\text { Empowerment through } \\
\text { contribution to research }\end{array}$ & $\begin{array}{l}\text { I think there would be a bit of a euphoria in terms of just simply participating in that direct way of the greater } \\
\text { effort of the project." (P13) }\end{array}$ \\
\hline $\begin{array}{l}\text { Sense of responsibility to the } \\
\text { choroideremia community (CHMers) }\end{array}$ & $\begin{array}{l}\text { "[Participating in a gene transfer clinical trial] would be a benefit, maybe not so much for me but for the } \\
\text { broader pool of CHMers. So I feel some sort of responsibility... for those of us who carry this disease." (P16) }\end{array}$ \\
\hline $\begin{array}{l}\text { Aid in development of therapy for } \\
\text { future generations }\end{array}$ & $\begin{array}{l}\text { "[Participating in a gene transfer clinical trial would be a benefit]... if the research is successful....and there is a } \\
\text { treatment...that would help future generations and other people that are suffering the same disease or similar } \\
\text { diseases." (P6) }\end{array}$ \\
\hline \multicolumn{2}{|l|}{ Direct benefits } \\
\hline Visual benefits & $\begin{array}{l}\text { "If there was a therapy...that would be a dream come true, that would be unbelievable having full sight now, } \\
\text { not having to worry about being blind in the future, being able to see at night which to me would be almost } \\
\text { like a superpower because I have never seen at night before. I would feel likes an X-Man if I could see at night!" } \\
\text { (P19) }\end{array}$ \\
\hline $\begin{array}{l}\text { Improved quality of life secondary to } \\
\text { visual benefits }\end{array}$ & $\begin{array}{l}\text { "...it's not just my vision. It's being able to do things that's associated with vision such as continued employment } \\
\text { and reduced dependency on friends and family and children." (P14) }\end{array}$ \\
\hline $\begin{array}{l}\text { Relief of anxiety secondary to visual } \\
\text { benefits }\end{array}$ & $\begin{array}{l}\text { "If they could stop it [vision loss] here, I'd feel like I won the lottery, because that's the fear. I know where I am. } \\
\text { I want to know where I am. And every time your eyes adjust... it's like changing a new world, going into a new } \\
\text { surrounding." (P17) }\end{array}$ \\
\hline
\end{tabular}


Table 2 Patient awareness of risks of harm associated with a phase I choroideremia gene transfer clinical trial

\begin{tabular}{ll} 
Risk category & Described by patients? \\
\hline Financial burdens & Some \\
\hline Psychological stress & Some \\
\hline Surgical risks & Some \\
\hline Germline gene transfer & No \\
\hline Loss of vision & Most \\
\hline Loss of an eye & No \\
\hline Insertional mutagenesis/oncogenesis & No \\
\hline Brain toxicitya & No \\
\hline Immune response to viral vector & Some \\
\hline Death & No \\
\hline
\end{tabular}

${ }^{a}$ Due to viral vector access to optic nerve.

tomorrow I'd have that procedure done, absolutely."-P2. But many patients worried that the time frame for clinical implementation of GT may not meet their own therapeutic window: "My eyes are degenerating. I can tell every month that there's less vision...I think there's urgency, and [GT] would allow me to keep more of my vision the sooner I get it."-P8. Others expressed frustration with the slow regulatory approval process: "The treatment is not available fast enough...There's a real sense of urgency...I know everybody wants to...do it...in careful way; but for me, I'd rather take the chance and save my eyes."-P20

Many patients explained that they would do anything to access GT: incur financial burdens; take time off of work; or travel. Some advocates echoed this perspective, revealing their personal stakes: "I will go for the second mortgage on my home if that's what it takes."-A1. Clinicians displayed an awareness of patients' urgency to access GT: "I think anybody...faced with the prospect of blindness [would] say that "I would do anything...to undertake clinical trial"...patients have said to me "I'd rather have a heart attack than lose my vision"'”-C9.

Time frames: "I know it's in sight, but will it be in my sight?" Uncertainty about time frames for the clinical implementation of GT was a major patient concern, particularly for patients who wanted to make practical arrangements for the future but did not know whether to accept their current prognosis and to plan for further vision loss or for the possibility of an intervention within a limited therapeutic window: "I don't even know the average time for any given clinical trial to go through the phases...Let's assume for example that the choroideremia [trial] phases are all successful, is it the best case scenario for something within a two-year time frame, five years, ten years, twenty years?"-P10.

Advocates were similarly frustrated with vague time frames presented by clinicians at fundraising venues: "It's difficult for [clinicians] because they're there to...generate optimism, because their goal is to generate funds... At the same time they have to be cautious about not raising hopes too high, and so you get the vague answer. Like, when will a certain thing be standard of care? It will become the five to eight year time frame." A2. Advocates were further disappointed and confused when projected time frames were not met: "Time frames in research never seem to be accurate... That's disappointing. It's difficult to understand."-A2.

Clinicians and advocates were aware of patient concerns surrounding time frames. "[Patients ask] when is [GT] going to be in the clinic?... So their main question is when are we going to see...the fruit of all of the research that has been done over the years that's going to-and they're blunt about it-cure the disease?" - PA3. Nevertheless, most clinicians did not communicate effectively about time frames, frustrating patients with vague or dismissive responses.

Left to interpret vague time frames, patients shared their estimates. Most patients hoped that GT would be available within their therapeutic window. However, some, who already had significantly deteriorated visual fields, understood that their therapeutic window had passed. These patients were hopeful that GT would provide visual benefits for future generations. For other patients, including affected advocates, the success of the LCA trials fueled hopes that adults, including themselves, will benefit from GT: "older guys...knew... research takes time, so...it's going to be too late for us...Then in the blink of an eye [following the LCA trials] ...that whole mindset did a complete 180 . Now there's a chance for my sight to be saved by a genetic therapy." $-\mathrm{A} 1$.

In terms of clinical implementation of GT, clinicians and advocates also expressed varying opinions about time frames. Most clinicians believed that treatment would be available for children. Most advocates also believed that GT would be available to children, some displaying a great deal of certainty in this prospect: "If a baby is born today, I have absolute full confidence that that baby is going to have a treatment before their eyes get so bad that there's going to be a noticeable difference in their sight." - A1

As clinicians and advocates quantified time frame estimates for the clinical implementation of GT, further ambiguities became apparent, with predictions ranging between 3 and 10 years. While some clinicians quantified their predictions, others made general remarks about the progress in the field or refused to provide estimates altogether. Clinicians explained that it is difficult to predict time frames, but some suggested informing patients about the phases of clinical trials and associated average time frames: "The one thing that I think would help...when they [patients] think about research [is]...explaining those different phases of a clinical trial" $-\mathrm{C} 2$

\section{Balancing risks of harm and potential benefits in light of uncertain time frames: the "two-pronged approach"}

Clinicians explained that communications must balance patient hopes for visual benefit with the uncertainties of early-stage GT trials and the current reality of limited clinical care. Clinicians identified a "two-pronged approach" to promote balanced communications about GT, which would enable clinicians to 
share in patient hope for a future treatment, while emphasizing the experimental nature of GT and associated risks of harm (Table 2). The approach would promote a discussion of both experimental options and disease management. A realistic representation of time frames may prepare patients for a prognosis of continued visual impairment if a treatment does not materialize within a limited therapeutic window. The two-pronged approach could distinguish between the theoretical promise of GT, affirming patient hopes, and the current clinical reality: "We cannot assume what [choroideremia GT] will look like in five years. So we can only talk about what we know. We can say where we hope to go, but be very clear that that's a wish, that is not reality right now."-C3.

\section{DISCUSSION}

The prospect of GT for choroideremia has fueled patient hopes for therapeutic benefit; provided a sense of urgency for its clinical implementation; and, in the interim, motivated patients to privilege considerations of potential benefits over risks of harm in anticipated clinical trial recruitment. Most participants cited qualified hopes for potential benefits rather than confident expectations. In other words, they remained optimistic without being able to express specific likelihoods for benefits ${ }^{15}$ and acknowledged the possibility that GT may not generate visual benefits. Similar to other studies, patient hope for direct benefits ${ }^{24-26}$ greatly surpassed aspirational and collateral benefits as a motivator for trial participation, ${ }^{27}$ a finding indicative of a therapeutic misconception. ${ }^{13}$

When stakeholders view CTs as both "science and a source of succor" ${ }^{28}$ or an opportunity for patient care, ${ }^{15,19}$ problems arise because there is a less than $1 \%$ chance for clinical improvement in phase I GT trials. ${ }^{29}$ Nevertheless, Lazarus-like responses have occurred in phase I trials, generating debate on the appropriateness of discussing direct benefits. In oncology trials, critics raise concerns about the use of surrogate measures (e.g., tumor response, stable disease), rather than end point measures (e.g., increased survival, improved quality of life). Surrogate measures may not be clinically meaningful and, at best, are suggestive of direct benefit. ${ }^{30}$

GT, as a novel and complex intervention, is at the forefront adapted trial design use, such as the use of secondary outcome measures. These may obscure the research-treatment distinction in early-phase trials, ${ }^{29}$ further confounding communications about the safety focus of phase I trials. Secondary outcome measures in ocular phase I GT trials (e.g., microperimetry, fundus imaging) confirm safety, but also measure potential efficacy. The LCA trials employed surrogate end point measures with significant shortcomings: end point measures for long-term safety and efficacy are still under development, ${ }^{9}$ as are measures for clinically meaningful improved visual function and quality of life. Although disputed by lead researchers of the LCA trials, ${ }^{31}$ a recent analysis indicates photoreceptor degradation continues after GT despite short-term improvements in visual function, raising concerns about long-term efficacy of GT. ${ }^{9}$ Other clinical trial design decisions, such as the inclusion of children in a phase I LCA trial to accommodate the limited therapeutic window of patients, ${ }^{7}$ further blurs the research-treatment distinction.

The implications of such adaptations to standard phase I clinical trial designs for the therapeutic hopes of patients ${ }^{32}$ and clinical investigators require further investigation. However, our results show that patients overestimated therapeutic benefit compared with other stakeholder groups. Ocular GT is not regenerative; it cannot revive degenerated photoreceptor cells ${ }^{23}$ and so cannot cure adult patients. Such therapeutic misestimation by patients has been observed in phase I oncology trials. ${ }^{33,34}$ Discrepancies may be due to heterogeneous interpretations of "benefit" from disease amelioration to cure. ${ }^{34}$

Therapeutic misestimation engages not only overestimation of potential benefits, but also underestimation risks of harm. ${ }^{14}$ As such, patient attention was diverted from risks of harm ${ }^{25}$ and directed toward gaining access to trials. Trust also mediated patient risk perspectives: patients revealed trust in science ${ }^{25}$ and trust in their physicians. ${ }^{27}$ Trust allows patients to reinterpret uncertainties in phase I trials as opportunities for potential benefits rather than as risks of harm. ${ }^{35}$

Despite prominent discussion of therapeutic benefits, uncertainties became apparent about the time frames associated with the clinical implementation of GT. "GT has often been characterized as permanently 5 years away from clinical application" ${ }^{36}$ Media portrayals of GT as an imminent cure, supported by positive quotations by leading researchers, reinforce this perspective. ${ }^{11}$ Overly optimistic hopes about time frames may not only lead to disappointment but also undermine the stated trust in researchers and threaten funding support. ${ }^{37}$ While predicting the future of ocular GT is impossible, the frustration of patients and advocates about uncertain or delayed time frames necessitates improved communication strategies. Research on communicating time frames, however, has focused primarily on communicating patient prognoses. ${ }^{38}$

\section{Recommendations and conclusion}

Communications of clinicians and advocacy organizations with patients must account for both therapeutic misconception and misestimation. The former demands a detailed explanation of the safety focus of early-stage clinical trials. However, the safety focus of phase I trials does not preclude the prospect of direct benefit. ${ }^{30}$ Communicators should therefore highlight that, while choroideremia GT could theoretically result in direct visual benefit, clinical equipoise exists given the lack of empirical data. Ensuring patient comprehension of the relevant risks of harm and uncertainties, qualifying incremental evidence in cases where medical benefits were observed in the LCA trials (i.e., measures of long-term efficacy and clinically meaningful improvement in quality of life are still under development), and emphasizing that aspirational and collateral benefits are more likely than direct medical benefits, are key strategies in promoting "informed hope" 39 among patients and informed consent for participation in GT trials. 
To address therapeutic misestimation, patient communications should be clear about the full spectrum of theoretically feasible visual outcomes from GT, in general, and in light of patientspecific therapeutic windows. Clinicians and advocates should be aware of the dominance of curative public discourse in the media and beyond and be prepared to counter such representations. ${ }^{11}$

Communicators should clearly situate the current state of choroideremia GT within the context of clinical research stages and time frames. As an exemplar, the LCA trials began in 2007, but are only now, in 2013, beginning to enroll patients in phase III studies (clinicaltrials.gov NCT00999609). Historical evidence suggests that it takes 10-14 years to move from novel target to drug approval. ${ }^{40}$ Novel biotechnologies, such as GT, given their unique risk profile and additional regulatory steps, may take even longer.

While grounded in realistic visual benefits and time frames, patient communicators should not downplay the hope raised by promising novel biotechnologies. A "two-pronged approach" to patient communications balances such hope with pragmatic discussion about strategies for living with progressive visual impairment. Patient advocacy organizations, in particular, should carefully balance their communications to raise funds for promising research against more pragmatic information on disease management, peer support, and access to credible information sources. ${ }^{1}$

Finally, patients demonstrated a more nuanced understanding of potential visual outcomes ${ }^{34}$ than suggested by clinicians. It is therefore important for other stakeholder groups to recognize patients as critically thinking experts, while acknowledging patient vulnerabilities. With this understanding, clinicians and patient advocacy organizations should focus on the quality of their communications in terms of presenting a balanced account of risks, benefits, and the state of research advances, rather than attempting to manage patient expectations. With these strategies, communicators may counter the sensationalism historically associated with GT, ${ }^{2,18,40}$ promote informed consent, and honor patient hope while grounding communications in current clinical realities.

\section{ACKNOWLEDGMENTS}

We thank Cindy Jardine and Judy Illes for providing feedback on the interview guides. We also thank Elaine Hyshka for reviewing the codebook and Janis Geary for providing valuable feedback on the discussion section. Finally, we thank the research participants who bravely shared their perspectives with us. This study was supported by a Canadian Institutes of Health Research (CIHR): Emerging Team Grant, Rare Diseases (I.M. and T.B.) funded by the Canadian Institutes of Health Research, Foundation Fighting Blindness Canada, Choroideremia Research Foundation Canada, and an Alberta Innovates Health Solutions-CRIO Team Grant (I.M. and T.B.). S.B. was supported by graduate student stipends from the University of Alberta (QEll Graduate Scholarship, Myer Horowitz Graduate Students' Association Graduate Scholarship, and Department of Ophthalmology Graduate Student Stipend), Alberta Advanced Education and Technology Graduate Scholarship, and a Health Quality Council of Alberta Studentship. The funders had no role in study design, data collection and analysis, decision to publish, or preparation of the manuscript.

\section{DISCLOSURE}

The authors declare no conflict of interest.

\section{REFERENCES}

1. Stockdale A. Waiting for the cure: mapping the social relations of human gene therapy research. Sociol Health IIIn 1999;21:579-596.

2. Branca MA. Gene therapy: cursed or inching towards credibility? Nat Biotechnol 2005;23:519-521.

3. Bainbridge JW, Smith AJ, Barker SS, et al. Effect of gene therapy on visual function in Leber's congenital amaurosis. N Engl J Med 2008;358:2231-2239.

4. Hauswirth WW, Aleman TS, Kaushal S, et al. Treatment of leber congenital amaurosis due to RPE65 mutations by ocular subretinal injection of adenoassociated virus gene vector: short-term results of a phase I trial. Hum Gene Ther 2008; 19:979-990.

5. Maguire AM, Simonelli F, Pierce EA, et al. Safety and efficacy of gene transfer for Leber's congenital amaurosis. N Engl J Med 2008;358:2240-2248.

6. Koenekoop RK. Successful RPE65 gene replacement and improved visual function in humans. Ophthalmic Genet 2008;29:89-91.

7. Maguire AM, High KA, Auricchio A, et al. Age-dependent effects of RPE65 gene therapy for Leber's congenital amaurosis: a phase 1 dose-escalation trial. Lancet 2009;374:1597-1605.

8. Héon E. My child has Leber congenital amaurosis: why is he/she not eligible for gene therapy trials? J AAPOS 2009;13:533-534.

9. Wojno AP, Pierce EA, Bennett J. Seeing the light. Sci Trans/ Med 2013;5:175fs8

10. MacDonald IM, Russell L, Chan CC. Choroideremia: new findings from ocular pathology and review of recent literature. Surv Ophthalmol 2009;54:401-407.

11. Benjaminy S. Ocular gene transfer communications: developing ethical frameworks for phase I choroideremia clinical trials [dissertation]. Department of Public Health Sciences, University of Alberta: Edmonton, Alberta, 2013.

12. Kimmelman J. Gene Transfer and the Ethics of First-in-Human Research: Lost in Translation. Cambridge University Press: Cambridge, UK; 2010.

13. Appelbaum PS, Roth LH, Lidz C. The therapeutic misconception: informed consent in psychiatric research. Int J Law Psychiatry 1982;5:319-329.

14. Horng S, Grady C. Misunderstanding in clinical research: distinguishing therapeutic misconception, therapeutic misestimation, and therapeutic optimism. IRB 2003;25:11-16.

15. Henderson GE, Davis AM, King NM, et al. Uncertain benefit: investigators' views and communications in early phase gene transfer trials. Mol Ther 2004;10:225231

16. Kent A, King NM, Cohen-Haguenauer O. Toward a proportionate regulatory framework for gene transfer: a patient group-led initiative. Hum Gene Ther 2011:22:126-134.

17. Illes J, Reimer JC, Kwon BK. Stem cell clinical trials for spinal cord injury: readiness, reluctance, redefinition. Stem Cell Rev 2011;7:997-1005.

18. Wilson JM. Medicine. A history lesson for stem cells. Science 2009;324:727728.

19. Henderson GE, Easter MM, Zimmer C, et al. Therapeutic misconception in early phase gene transfer trials. Soc Sci Med 2006;62:239-253.

20. McAllister M, Davies L, Payne K, Nicholls S, Donnai D, MacLeod R. The emotional effects of genetic diseases: implications for clinical genetics. Am J Med Genet A 2007;143A:2651-2661.

21. Charmaz K. Constructing Grounded Theory: A Practical Guide through Qualitative Analysis. Sage Publications: Thousand Oaks, CA; 2006.

22. King NM. Defining and describing benefit appropriately in clinical trials. J Law Med Ethics 2000;28:332-343.

23. Jacobson SG, Aleman TS, Cideciyan AV, et al. Identifying photoreceptors in blind eyes caused by RPE65 mutations: Prerequisite for human gene therapy success. Proc Natl Acad Sci USA 2005;102:6177-6182.

24. Kwon BK, Ghag A, Dvorak MF, Tetzlaff W, Illes J. Expectations of benefit and tolerance to risk of individuals with spinal cord injury regarding potential participation in clinical trials. J Neurotrauma 2012;29:2727-2737.

25. Pentz RD, White M, Harvey RD, et al. Therapeutic misconception, misestimation, and optimism in participants enrolled in phase 1 trials. Cancer 2012;118:45714578.

26. Miller VA, Baker JN, Leek AC, et al. Adolescent perspectives on phase I cancer research. Pediatr Blood Cancer 2013;60:873-878. 
27. Sulmasy DP, Astrow AB, He MK, et al. The culture of faith and hope: patients' justifications for their high estimations of expected therapeutic benefit when enrolling in early phase oncology trials. Cancer 2010;116:3702-3711.

28. Evans R, Kotchetkova I, Langer S. Just around the corner: rhetorics of progress and promise in genetic research. Public Underst Sci 2009:18:43-59.

29. Kimmelman J. Ethics of cancer gene transfer clinical research. Methods Mol Biol 2009:542:423-445

30. Miller FG, Joffe S. Phase 1 oncology trials and informed consent. J Med Ethics e-pub ahead of print 17 November, 2012. doi:10.1136/medethics-2012-100832.

31. Cideciyan $A V$, Jacobson $S G$, Beltran WA, et al. Human retinal gene therapy for Leber congenital amaurosis shows advancing retinal degeneration despite enduring visual improvement. Proc Natl Acad Sci USA 2013;110:E517-E525.

32. Hyun I. Therapeutic hope, spiritual distress, and the problem of stem cell tourism. Cell Stem Cell 2013;12:505-507.

33. Cheng JD, Hitt J, Koczwara B, et al. Impact of quality of life on patient expectations regarding phase I clinical trials. J Clin Oncol 2000;18:421-428.

34. Weinfurt KP, Castel LD, Li Y, et al. The correlation between patient characteristics and expectations of benefit from Phase I clinical trials. Cancer 2003;98:166175.
35. Kim SY, Holloway RG, Frank S, Wilson R, Kieburtz K. Trust in early phase research: therapeutic optimism and protective pessimism. Med Health Care Philos 2008:11:393-401.

36. Kimmelman J. The ethics of human gene transfer. Nat Rev Genet 2008;9:239244.

37. Petersen $A$. The ethics of expectations: biobanks and the promise of personalised medicine. Monash Bioeth Rev 2009;28:5.1-512.

38. Innes $S$, Payne S. Advanced cancer patients' prognostic information preferences: a review. Palliat Med 2009;23:29-39.

39. Reimer J, Borgelt E, Illes J. In pursuit of "informed hope" in the stem cell discourse. Am J Bioeth 2010:10:31-32.

40. Glassman RH, Sun AY. Biotechnology: identifying advances from the hype. Nat Rev Drug Discov 2004;3:177-183.

(i) (\$) This work is licensed under a Creative Commons Attribution-NonCommercial-NoDerivative Works 3.0 Unported License. To view a copy of this license, visit http://creativecommons.org/licenses/by-nc-nd/3.0/ 